\title{
POTENCIAL FISIOLÓGICO DE SEMENTES DE MOGANGO E DESEMPENHO DAS PLANTAS NO CAMPO'
}

\author{
PAULA FERREIRA VAZ DE ÁVILA MALONE², FRANCISCO AMARAL VILLELA³, CARLOS ROGÉRIO MAUCH4.
}

\begin{abstract}
RESUMO - O objetivo desse estudo foi comparar a eficiência de diferentes testes de vigor na avaliação da qualidade fisiológica em sementes de mogango, relacionando os resultados obtidos em laboratório com o desempenho das plantas no campo. Os lotes foram avaliados pelos testes de germinação, primeira contagem, envelhecimento acelerado tradicional e com uso de solução salina não saturada de $\mathrm{NaCl}$, (ambos a $41^{\circ} \mathrm{C} / 48$ e $72 \mathrm{~h}$ ) e comparados com a emergência e o desenvolvimento inicial das plantas no campo. As plantas foram avaliadas aos quinze, vinte cinco e trinta e cinco dias após a semeadura. Foram determinados o comprimento de parte aérea e de raiz, número de folhas e massa seca de planta. O delineamento experimental foi inteiramente casualizado, utilizando-se quatro tratamentos (lotes) com quatro repetições. Conclui-se que o teste de envelhecimento acelerado tradicional por $72 \mathrm{~h}$ permitiu classificar os lotes em diferentes níveis de vigor, e é o mais adequado para avaliação do potencial fisiológico de sementes de mogango. Diferenças acentuadas quanto de vigor entre lotes de sementes de mogango influenciaram o desempenho inicial da cultura, especialmente o comprimento da parte aérea e a matéria seca da planta.
\end{abstract}

Termos para Indexação: Cucurbita pepo, vigor, estabelecimento de plantas.

\section{MOGANGO PHYSIOLOGICAL SEED POTENTIAL AND FIELD PERFORMANCE}

\begin{abstract}
The objective of this study was to compare different vigor tests used to evaluate physiological quality in mogango seeds, and the relationship between the results obtained in the laboratory and field performance. The lots were evaluated by the following tests: standard germination, first count of germination, traditional accelerated aging and with non-saturated $\mathrm{NaCl}$ solution (both at $41^{\circ} \mathrm{C} / 48$ and $72 \mathrm{~h}$ ) and compared with emergence and plant development. The plants were evaluated at fifteen, twenty five and thirty five days after sowing. Plant height, number of leaves and dry weight were estimated. A randomized complete block design was used with four treatments (lots) and four replications. Based on the results it was concluded that traditional $72 \mathrm{~h}$ accelerated aging provided a better differentiation among mogango seed lots with different physiological quality. Variations in mogango seed lots can affect the initial plant performance, especially plant height and dry weight.
\end{abstract}

Index terms: Cucurbita pepo, vigor, plant establishment.

Submetido em 12/10/2007. Aceito para publicação em 28/04/2008. ${ }^{2}$ Eng $^{\mathrm{a}}$ Agr $^{\mathrm{a}}$. Doutoranda do Programa de Pós-Graduação em AgronomiaProdução Vegetal, UFPel/FAEM. Caixa Postal 354, 96010-900. Pelotas/ RS. ${ }^{3} E^{\circ}{ }^{o}$ Agrícola, Dr..Prof. do Programa de Pós-Graduação em Ciência e Tecnologia de Sementes, UFPel/FAEM, Caixa Postal 354, 96010-900. Pelotas/RS. ${ }^{4} \mathrm{Eng}^{\mathrm{O}} \mathrm{Agr}{ }^{\mathrm{O}}$. Dr. Prof. do Departamento de Fitotecnia , UFPel/ FAEM, Caixa Postal 354, 96010-900. Pelotas/RS. 


\section{INTRODUÇÃO}

O mogango (Cucurbita pepo L.) pertence à família das cucurbitáceas. No Brasil, é muito cultivado no estado do Rio Grande do Sul, sendo muito apreciado pela culinária gaúcha. A propagação do mogango é feita por sementes que podem ser semeadas diretamente no local de cultivo ou em bandejas com células individuais para posterior transplantio, sendo indispensável à utilização de sementes com elevado vigor.

A qualidade final de um produto olerícola depende entre outros fatores, da obtenção de uma população adequada e uniforme de plantas no campo. A emergência de plântulas no campo pode variar, mesmo para lotes de sementes com alta percentagem de germinação, em função do vigor das sementes. Estudos sobre o vigor de sementes são importantes para a agricultura, pois permitem a obtenção de estimativas do potencial fisiológico das sementes, com a identificação de diferenças significativas entre lotes, geralmente, não detectadas pelo teste de germinação (Ramos et al.; 2004).

Os principais objetivos da pesquisa sobre o vigor de sementes são o desenvolvimento de procedimentos confiáveis para avaliá-lo e determinar sua influência sobre o desempenho das plântulas e plantas em campo. Há várias referências na literatura que indicam associação consistente entre o potencial fisiológico das sementes, determinado em laboratório, e a emergência das plântulas em campo. No entanto, o mesmo não acontece com a relação do potencial fisiológico com o desempenho das plantas e com a produção final (Kikuti e Marcos Filho, 2007).

As relações entre o vigor das sementes e o desempenho das plantas em campo também não estão suficientemente esclarecidas, tanto no Brasil como no exterior. Há consenso quanto à influência do vigor sobre a emergência das plântulas e o desenvolvimento inicial das plantas, mas não se conhece perfeitamente até que ponto esses efeitos se estendem até estádios fenológicos mais avançados e afetam a produção da cultura (Ellis, 1992; Marcos Filho, 2005).

Diversos são os testes de vigor relatados na literatura e que têm sido indicados para sementes de hortaliças. A primeira contagem da germinação, realizada normalmente para facilitar a condução do teste de germinação, pode ser considerada um teste de vigor, pois, sabe-se que no processo de deterioração, a velocidade de germinação decai antes da porcentagem de germinação. Assim, as amostras que germinam mais rapidamente, apresentando valores mais elevados de germinação na primeira contagem, podem ser consideradas mais vigorosas que aquelas de germinação mais lenta (Matthews, 1980).
Outro teste bastante utilizado é o envelhecimento acelerado, que foi desenvolvido inicialmente como um método para estimar o potencial de armazenamento, mostrouse eficiente para classificar lotes segundo sua capacidade de estabelecimento no campo. Nesse teste, a velocidade dos processos deteriorativos é acelerada, expondo-se as sementes a níveis elevados de temperatura e umidade relativa do ar (Spinola et al; 1998), baseando-se no fato de que a taxa de deterioração das sementes aumenta consideravelmente quando submetidas a tais condições (Marcos Filho, 1999). Segundo este autor, o teste pode ser considerado como um dos mais sensíveis para a avaliação do vigor, dentre os disponíveis na atualidade. No entanto, seu uso em sementes de hortaliças ainda é restrito. Piana et al. (1995) verificaram que o teste de envelhecimento acelerado foi um dos que mais se relacionou à emergência das plântulas de cebola em campo, além de identificar lotes com diferentes níveis de vigor. Resultados semelhantes foram obtidos com sementes de cenoura (Trigo e Trigo, 1995b), pepino (Trigo e Trigo, 1995a), brócolos (Mello et al., 1999) e tomate (Panobianco e Marcos Filho, 2001).

Estudos conduzidos com a cultura de mogango não foram encontrados na bibliografia principalmente quanto à tecnologia de sementes, assim como o número de trabalhos dirigidos à comparação entre os resultados dos testes de vigor e os de emergência das plântulas no campo. Desta forma, é desejável a obtenção de mais informações que permitam identificar lotes que possuam maiores possibilidades de apresentar desempenho superior em condições de campo, ou seja, avaliar corretamente o potencial fisiológico das sementes de cada lote. Com essas colocações, o objetivo desse estudo foi comparar a eficiência de testes de vigor para avaliação da qualidade fisiológica das sementes de mogango, relacionando os resultados obtidos em laboratório com seu o desempenho das plantas no campo.

\section{MATERIAL E MÉTODOS}

A pesquisa foi conduzida no Laboratório Didático de Análise de Sementes do Departamento de Fitotecnia, Faculdade de Agronomia "Eliseu Maciel", Universidade Federal de Pelotas, em Pelotas-RS. Foram utilizados quatro lotes de sementes de mogango, cultivar Sul Mineiro. A qualidade inicial das sementes foi avaliada pelos seguintes testes e/ou determinações:

Teor de água - realizado com duas subamostras de quatro a cinco gramas de sementes para cada lote, pelo método da estufa $105 \pm 3^{\circ} \mathrm{C}$, durante 24 horas, de acordo com 
as Regras para Análise de Sementes-RAS (Brasil, 1992). Os resultados foram expressos em porcentagem.

Germinação - conduzido com oito subamostras de 50 sementes, constituindo quatro repetições de cem sementes para cada lote, distribuídas entre três folhas de papel germitest, umedecidas com água destilada, na proporção de 2,5 vezes o peso o papel seco. Após a semeadura, foram feitos rolos, que foram mantidos em germinador a $25^{\circ} \mathrm{C}$. As avaliações foram realizadas no quarto e oitavo dia após a semeadura, conforme as RAS (Brasil, 1992), computando-se as porcentagens de plântulas normais.

Primeira contagem da germinação - realizada conjuntamente com o teste de germinação, obtendo-se a porcentagem de plântulas normais no quarto dia após instalação do teste.

Envelhecimento acelerado tradicional - conduzido com a utilização de caixas plásticas tipo gerbox, com compartimento individual (minicâmaras), contendo $40 \mathrm{~mL}$ de água, uma bandeja de tela de alumínio, onde as sementes foram distribuídas formando uma camada uniforme. As caixas foram mantidas em câmara durante 48 e $72 \mathrm{~h}$ a $41^{\circ} \mathrm{C}$. Decorrido cada período de envelhecimento, 16 subamostras de 50 sementes por lote foram submetidas ao teste de germinação, seguindo metodologia descrita anteriormente, com avaliação realizada no quarto dia após a semeadura. Paralelamente, foi determinado o teor de água das sementes após cada período de envelhecimento, para verificar a uniformidade das condições do teste, conforme Marcos Filho (1999).

Envelhecimento acelerado com uso de solução salina não saturada - conduzido utilizando metodologia similar ao envelhecimento acelerado tradicional, porém adicionando-se ao fundo da caixa plástica, $40 \mathrm{~mL}$ de solução salina $(11 \mathrm{~g}$ de $\mathrm{NaCl}$ em $100 \mathrm{~mL}$ ), estabelecendo um ambiente com umidade relativa de 94\%, conforme Ávila et al. (2006).

Emergência de plântulas em campo - determinada com oito subamostras de 50 sementes por lote, distribuídas em canteiros, com um espaçamento de $10 \mathrm{~cm}$ entre sementes, sob temperatura ambiente em torno de $25^{\circ} \mathrm{C}$. As avaliações foram realizadas mediante contagem do número de plântulas emergidas no décimo quarto dia após a semeadura. Foi calculada a porcentagem de emergência de plântulas, conforme Nakagawa, (1999).

Análise das plantas em campo: A avaliação das plantas em campo foi realizada aos quinze, vinte cinco e trinta e cinco dias após a semeadura, coletando-se 10 plantas por repetição de cada lote, para as seguintes determinações:

Comprimento médio da parte aérea - expresso em centímetros, mediante a medição do comprimento entre o colo e o ápice da última folha de cada planta da amostra;

Comprimento médio de raiz - expresso em centímetros, tomado do colo até o ápice da raiz principal de cada planta da amostra;

Número médio de folhas - realizado através da contagem do número de folhas de cada uma das plantas amostradas;

Matéria seca - realizada nas dez plantas amostradas por repetição, sendo essas colocadas em sacos de papel e submetidas à secagem em estufa a $70^{\circ} \mathrm{C}$, até peso constante, seguida por pesagem em balança analítica, com precisão de $0,001 \mathrm{~g}$.

\section{Procedimento estatístico}

O delineamento experimental foi inteiramente casualizado, empregando-se quatro tratamentos (lotes) e quatro repetições. As médias foram comparadas pelo teste de Tukey a 5\% de probabilidade. Para os cálculos estatísticos utilizou-se o Sistema de Análise Estatística SASM-Agri (Canteri et al. 2001). Cada procedimento (combinação de solução colocada no fundo das caixas plásticas e de período de exposição) para o teste de envelhecimento acelerado foi analisado separadamente, sendo os lotes considerados tratamentos. Os dados de teor de água das sementes não foram submetidos à análise estatística.

\section{RESULTADOS E DISCUSSÃO}

Os resultados referentes à qualidade inicial dos lotes de sementes de mogango encontram-se na tabela 1 .

Pode-se observar pelo teste de germinação que os lotes A, C e D, apresentaram germinação semelhante e superiores ao lote B. dados esses também observados na primeira contagem da germinação. Embora a primeira contagem da germinação possa ser considerada um indicativo de vigor, sabe-se que a redução da velocidade de germinação não está incluída entre os eventos iniciais do processo de deterioração de sementes (Delouche e Baskin, 1973), ocorrência que pode justificar a baixa sensibilidade desse teste para detectar diferenças menos acentuadas de vigor e os conseqüentes agrupamentos dos demais lotes em único nível de vigor.

$\mathrm{Na}$ análise dos resultados do teste de envelhecimento acelerado, pode-se observar uma variação entre períodos de envelhecimento e métodos utilizados, podendo-se destacar o método tradicional com período de $72 \mathrm{~h}$ de envelhecimento, o qual apresentou resultados semelhantes à emergência em campo. Torres e Marcos Filho (2003) verificaram que o teste de envelhecimento acelerado utilizando a combinação 41 
${ }^{\circ} \mathrm{C}$ por 72 horas, tanto pelo método tradicional como pelo alternativo (com solução de $\mathrm{NaCl}$ ), apresentou sensibilidade suficiente para a avaliação da qualidade fisiológica de sementes de melão. Desta forma pode-se salientar que a utilização de solução salina de $\mathrm{NaCl}$, justifica-se apenas para hortaliças com sementes de tamanho relativamente pequeno, pois o método tradicional possibilita que as sementes absorvam muita água e não consigam assim promover estresse adequado às sementes para separar os lotes em diferentes níveis de vigor.

TABELA 1. Qualidade inicial de quatro lotes (A, B, C e D) de sementes de mogango avaliada pelos testes de germinação (TG), primeira contagem da germinação (PCG), envelhecimento acelerado tradicional por $48 \mathrm{~h}$ e $72 \mathrm{~h}$ (EAT48H e EAT72H) e com utilização de solução salina por 48h e 72h (EAS48h e EAS72h) e emergência de plântulas (EP). Pelotas-RS, 2007.

\begin{tabular}{llllllll}
\hline Lotes & TG & PCG & EAT48H & EAS48H & EAT72H & EAS72H & EP \\
\hline A & $94 \mathrm{a}$ & $88 \mathrm{a}$ & $85 \mathrm{a}$ & $80 \mathrm{~b}$ & $78 \mathrm{~b}$ & $74^{\mathrm{a}}$ & $79 \mathrm{~b}$ \\
$\mathrm{~B}$ & $84 \mathrm{~b}$ & $67 \mathrm{~b}$ & $56 \mathrm{c}$ & $55 \mathrm{c}$ & $57 \mathrm{c}$ & $48 \mathrm{c}$ & $72 \mathrm{c}$ \\
$\mathrm{C}$ & $94 \mathrm{a}$ & $91 \mathrm{a}$ & $84 \mathrm{a}$ & $80 \mathrm{~b}$ & $90 \mathrm{a}$ & $73 \mathrm{a}$ & $85 \mathrm{a}$ \\
$\mathrm{D}$ & $96 \mathrm{a}$ & $87 \mathrm{a}$ & $79 \mathrm{~b}$ & $88 \mathrm{a}$ & $84 \mathrm{~b}$ & $58 \mathrm{~b}$ & $82 \mathrm{ab}$ \\
\hline
\end{tabular}

Médias seguidas de mesma letra na coluna, não diferem entre si em nível de $5 \%$ de probabilidade, pelo teste de Tukey

Estes resultados do teste de envelhecimento acelerado tradicional por $72 \mathrm{hs}$ destacam o lote $\mathrm{C}$ como superior, $\mathrm{B}$ como inferior e os lotes A e D como intermediários, conforme a separação dos lotes obtida na avaliação de emergência em campo .

O teste de envelhecimento acelerado foi um dos que mais se relacionou com a emergência de plântulas de cebola no campo, além de identificar lotes com diferentes níveis de vigor (Piana et al., 1995). Fato esse também verificado neste estudo. Resultados semelhantes foram encontrados em trabalhos com sementes de pepino (Trigo e Trigo, 1995 a), brócolis (Mello et al., 1999 e Mendonça et al., 2000), tomate (Panobianco e Marcos Filho, 2001) e melancia (Bhering et al., 2003). Pode-se observar na tabela 2, os resultados de teor de água inicial e após a realização do teste de envelhecimento acelerado tradicional e com uso de solução salina não saturada de $\mathrm{NaCl}$, evidenciando que o uso da solução salina promove redução da captação de água pelas sementes de mogango durante o período de envelhecimento, de modo que ao final do teste as sementes atingiram teores de água sensivelmente inferiores aos verificados com a utilização do método tradicional. Nesse caso por ser o mogango uma espécie com sementes de tamanho relativamente grande, a solução salina não saturada não causou estresse suficiente que permitisse a separação dos lotes em níveis de vigor. Por outro lado, foi possível observar outro aspecto interessante da utilização dos tratamentos com $\mathrm{NaCl}$, que, após os períodos de envelhecimento, não houve a proliferação de microrganismos nas sementes, observação está já elucidada por Ávila et al. (2006) e por vários autores, com utilização de solução saturada de sal, sendo esta considerada uma vantagem dessa metodologia.

TABELA 2. Teores de água (\%) inicial e após os períodos de envelhecimento acelerado tradicional (H2O) e com solução salina $(\mathrm{NaCl})$ das sementes de quatro lotes de mogango. Pelotas-RS, 2007.

\begin{tabular}{cccccc}
\hline Lotes & Inicial & $\mathrm{H}_{2} \mathrm{O} 48 \mathrm{~h}$ & $\mathrm{H}_{2} \mathrm{O} 72 \mathrm{~h}$ & $\mathrm{NaCl} 48 \mathrm{~h}$ & $\mathrm{NaCl} 72 \mathrm{~h}$ \\
\hline $\mathrm{A}$ & 4,9 & 18,0 & 19,4 & 12,3 & 11,7 \\
$\mathrm{~B}$ & 4,4 & 18,1 & 18,2 & 11,3 & 11,8 \\
$\mathrm{C}$ & 5,2 & 18,9 & 19,5 & 12,8 & 12,4 \\
$\mathrm{D}$ & 4,9 & 17,2 & 17,2 & 12,7 & 10,8 \\
\hline
\end{tabular}


Nas tabelas 3, 4 e 5 estão expostos os dados referentes ao desempenho das plantas com quinze, vinte e cinco e trinta e cinco dias.

TABELA3. Resultados obtidos da análise de comprimento (cm) da parte aérea (PA), e raiz $(\mathrm{R})$, número de folhas (NF) e massa seca (g) de planta (MS) aos quinze dias após a semeadura. Pelotas-RS, 2007.

\begin{tabular}{ccccc}
\hline Lotes & $\mathrm{PA}$ & $\mathrm{R}$ & $\mathrm{NF}$ & $\mathrm{MS}$ \\
\hline $\mathrm{A}$ & $14,0 \mathrm{~b}$ & $7,3 \mathrm{ab}$ & $2,0 \mathrm{a}$ & $3,5 \mathrm{~b}$ \\
$\mathrm{~B}$ & $14,0 \mathrm{~b}$ & $7,0 \mathrm{~b}$ & $2,0 \mathrm{a}$ & $3,8 \mathrm{~b}$ \\
$\mathrm{C}$ & $16,8 \mathrm{a}$ & $7,8 \mathrm{ab}$ & $2,0 \mathrm{a}$ & $5,1 \mathrm{a}$ \\
$\mathrm{D}$ & $15,8 \mathrm{ab}$ & $9,5 \mathrm{a}$ & $2,0 \mathrm{a}$ & $4,9 \mathrm{a}$ \\
\hline
\end{tabular}

Médias seguidas de mesma letra na coluna, não diferem entre si em nível de $5 \%$ de probabilidade, pelo teste de Tukey.

TABELA4 . Resultados obtidos da análise de comprimento $(\mathrm{cm})$ da parte aérea $(\mathrm{PA})$, e raiz $(\mathrm{R})$, número de folhas (NF) e massa seca (g) de planta (MS) aos vinte e cinco dias após a semeadura. Pelotas-RS, 2007.

\begin{tabular}{ccccc}
\hline Lotes & PA & R & NF & MS \\
\hline A & $22,0 \mathrm{~b}$ & $15,3 \mathrm{a}$ & $6 \mathrm{a}$ & $14,9 \mathrm{~b}$ \\
$\mathrm{~B}$ & $18,8 \mathrm{c}$ & $10,8 \mathrm{c}$ & $5 \mathrm{~b}$ & $14,0 \mathrm{c}$ \\
$\mathrm{C}$ & $25,5 \mathrm{a}$ & $15,0 \mathrm{a}$ & $6 \mathrm{a}$ & $16,0 \mathrm{a}$ \\
$\mathrm{D}$ & $23,8 \mathrm{ab}$ & $14,3 \mathrm{a}$ & $6 \mathrm{a}$ & $15,2 \mathrm{ab}$ \\
\hline
\end{tabular}

Médias seguidas de mesma letra na coluna, não diferem entre si em nível de $5 \%$ de probabilidade, pelo teste de Tukey.

TABELA5. Resultados obtidos da análise de comprimento $(\mathrm{cm})$ da parte aérea $(\mathrm{PA})$, e raiz $(\mathrm{R})$, número de folhas (NF) e massa seca (g) de planta (MS) aos trinta e cinco dias após a semeadura. Pelotas-RS, 2007.

\begin{tabular}{|c|c|c|c|c|}
\hline Lotes & PA & $\mathrm{R}$ & $\mathrm{NF}$ & MS \\
\hline A & $42,3 \quad \mathrm{c}$ & $12,0 \mathrm{~b}$ & $11,5 \mathrm{a}$ & $29,0 \quad \mathrm{c}$ \\
\hline B & $39,8 \mathrm{c}$ & $10,3 \mathrm{~b}$ & $9,3 \mathrm{~b}$ & $26,6 \mathrm{~d}$ \\
\hline $\mathrm{C}$ & $46,3 \mathrm{~b}$ & $15,0 \mathrm{a}$ & $11,5 \mathrm{a}$ & $35,4 \mathrm{a}$ \\
\hline D & $51,8 \mathrm{a}$ & $15,0 \mathrm{a}$ & $11,3 \mathrm{a}$ & $31,8 \mathrm{~b}$ \\
\hline
\end{tabular}

Médias seguidas de mesma letra na coluna, não diferem entre si em nível de $5 \%$ de probabilidade, pelo teste de Tukey.
A análise da tabela 3 permite verificar que dados do comprimento da parte aérea e matéria seca destacaram os lote $\mathrm{C}$, como de vigor mais elevado, dados já verificados nos testes de envelhecimento acelerado tradicional por $72 \mathrm{~h} \mathrm{e}$ emergência em campo. $\mathrm{O}$ número de folhas e o comprimento de raiz destacam a inferioridade do lote $\mathrm{B}$.

$\mathrm{O}$ vigor das sementes pode proporcionar um impulso inicial ao crescimento das plântulas, mas a extensão desses efeitos não está completamente esclarecida (Ellis, 1992). É provável que as diferenças no potencial fisiológico das sementes, identificadas em laboratório, não tenham sido amplas ao ponto de afetar o desempenho inicial das plantas no campo. De acordo com TeKrony e Egli (1991), desde que as plantas tenham ultrapassado um nível mínimo de desenvolvimento, o vigor das sementes não afetaria a produção.

$\mathrm{Na}$ tabela 4 verifica-se que os dados referentes às avaliações realizadas aos vinte cinco dias após a semeadura, constatando-se melhor separação dos lotes em níveis de vigor nas variáveis comprimento de parte aérea e matéria seca de planta, destacando os lotes $\mathrm{C}$ como superior, seguido do lote $\mathrm{A}$ e $\mathrm{D}$ e lote $\mathrm{B}$ como de vigor inferior aos demais, concordando com a classificação de lotes apresentados no teste de emergência em campo. Ainda mostra que as variáveis, número de folhas e comprimento de raiz, apenas separa o lote $\mathrm{B}$ de vigor inferior, conforme observado no comprimento de raiz e aos quinze dias e nos teste de germinação e primeira contagem.

Aos trinta e cinco dias após a semeadura (Tabela 5), o comprimento da parte aérea, de raiz e matéria seca evidenciam a superioridade dos lotes $\mathrm{C}$ e $\mathrm{D}$ e, no número de folhas, a inferioridade do lote $\mathrm{B}$.

Estudando o potencial fisiológico de sementes de couveflor e comparando com o desempenho em campo, Kikuti e Marcos Filho (2007) também concluíram que existe influência no desenvolvimento inicial das plantas quando essas apresentam diferenças acentuadas entre os lotes, como foi verificado nesse estudo, onde o lote $\mathrm{C}$ se apresentou superior na maioria dos resultados obtidos, bem como o lote B mostrou desempenho inferior entre os lotes analisados.

Essa observação não foi tão evidente no presente trabalho, pois aos quinze dias as plantas apresentaram distinção menos evidente no vigor dos lotes, do que aos vinte e cinco e trinta e cinco dias, quando foi possível observar com melhor clareza a separação dos lotes em níveis de vigor.

Trabalhando com sementes de rabanete, Marcos Filho e Kikuti (2006) enfatizam que de maneira geral, os resultados obtidos permitem afirmar que o uso de sementes vigorosas 
é justificável para assegurar o estabelecimento adequado do estande, mesmo que não haja resposta consistente em termos de produção final das plantas.

\section{CONCLUSÕES}

O teste de envelhecimento acelerado permite classificar lotes de sementes de mogango em diferentes níveis de vigor e dentre os procedimentos adotados, o método tradicional com uso de água com período de exposição de 72 horas, a $41^{\circ} \mathrm{C}$, é adequado para avaliação do potencial fisiológico de sementes de mogango.

Diferenças acentuadas quanto ao vigor entre lotes de sementes de mogango influenciam o desempenho inicial da cultura, especialmente o comprimento da parte aérea e a matéria seca da planta.

\section{REFERÊNCIAS}

ÁVILA, P.F.V.; VILlELA, F.A.; ÁVILA, M.S.V. Teste de envelhecimento acelerado para avaliação do potencial fisiológico de sementes de rabanete. Revista Brasileira de Sementes, Pelotas, v.28, n.3, p.52-58, 2006.

BHÉRING, M.C.; DIAS, D.C.F.S.; GOMES, J.M.; BARROS, D.I. Métodos para avaliação do vigor de sementes de pepino. Revista Brasileira de Sementes, v.22, n.2, p.171-175, 2000 .

BHÉRING, M.C.; DIAS, D.C.F.S.; BARROS, D.I.; DIAS, L.A.S.; TOKUNISA, D.A. Avaliação do vigor de sementes de melancia (Citrillus lanatus Schrad) pelo teste de envelhecimento acelerado. Revista Brasileira de Sementes, Pelotas, v.25, n.2, p.1-6, 2003.

BRASIL. Ministério da Agricultura e Reforma Agrária. Regras para análise de sementes. Brasília, DF: SNDAV/ DNDV/CLAV 1992. 365p.

CANTERI, M. G.; ALTHAUS, R. A.; VIRGENS FILHO, J. S.; GIGLIOTI, E. A.; GODOY, C. V. SASM - Agri : Sistema para análise e separação de médias em experimentos agrícolas pelos métodos Scoft - Knott, Tukey e Duncan. Revista Brasileira de Agrocomputação, v.1, n.2, p.18-24. 2001.

DELOUCHE, J.C.; BASKIN, C.C. Accelerated aging techniques for predicting the relative storability of seed lots. Seed Science and Technology, Zürich, v.1, n.2, p.427-452, 1973.

ELLIS, R.H. Seed and seedling vigor in relation to growth and yield. Plant Growth Regulation, v.11, n.1, p. 249-255,
1992.

KIKUTI, A.L.P.; MARCOS FILHO, J. Potencial fisiológico de sementes de couve-flor e desempenho de plantas em campo. Revista Brasileira de Sementes, Pelotas, v.29, n1, p.107-113, 2007.

MARCOS FILHO, J. Teste de envelhecimento acelerado. In: KRZYZANOWSKI, F.C.; VIEIRA, R.D.; FRANÇA NETO, J.B. (Ed.). Vigor de sementes: conceitos e testes. Londrina: ABRATES, 1999. p.3.1-3.24.

MARCOS FILHO, J; KIKUTI, A.L.P. Vigor de sementes de rabanete e o desempenho de plantas em campo. Revista Brasileira de Sementes, Pelotas, v.28, n3, p.44-51, 2006.

MARCOS FILHO, J. Fisiologia de sementes de plantas cultivadas. Piracicaba: FEALQ, 2005. 495p.

MATTHEWS, S. Controlled deterioration: A new vigour test for crop seeds. In: HABBLETHWAITE, P.D. Seed production. London: Butterworths. 1980. p.647-660.

MELLO, S.C.; SPINOLA, M.C.M.; MINAMI, K. Métodos de avaliação da qualidade fisiológica de sementes de brócolos. Scientia Agricola, Piracicaba, v.56, n.4, p.1151$1155,1999$.

MENDONÇA, E.A.F.; RAMOS, N.P.; FESSEL, S.A.; SADER, R. Teste de deterioração controlada em sementes de brócolis. Revista Brasileira de Sementes, Pelotas, v.22, n.2, p.280-287, 2000.

NAKAGAWA, J. Testes de vigor baseados no desempenho das plântulas. In: KRZYZANOWSKI, F.C.; VIEIRA, R.D.; FRANÇA NETO, J.B. (Ed.). Vigor de sementes: conceitos e testes. Londrina: ABRATES, 1999. p.2.1-2.24.

PANOBIANCO, M.; MARCOS FILHO, J. Evaluation of the physiological potential of tomato seeds by germination and vigor tests. Seed Technology, v.23, n.2, p.151-161, 2001.

PIANA, Z.; TILLMANN, M.A.A.; MINAMI, K. Avaliação da qualidade fisiológica de sementes de cebola e sua relação com a formação de mudas vigorosas. Revista Brasileira de Sementes, Brasília, DF, v.17, n.2, p.149-153, 1995.

RAMOS, N.P.; FLOR, E.P.O.; MENDONÇA, E.A.F.; MINAMI, K. Envelhecimento acelerado em sementes de rúcula (Eruca sativa L.). Revista Brasileira de Sementes, Pelotas, v.26, n.1, p.98-103, 2004.

SPINOLA, M.C.M.; CALLIARI, M.F.; MARTINS, L.; TESSARIOLI NETO, J. Comparação entre métodos para avaliação do vigor de sementes de cenoura. Revista Brasileira de Sementes, Londrina, v.20, n.2, p.301-305, 1998. 
TeKRONY, D.M.; EGLI, D.B. Relationship of seed vigor to crop yield. Crop Science, Madison, v.31, n.3, p.816-822, 1991.

TORRES, S.B \& MARCOS FILHO, J. Accelerated aging of melon seeds. Scientia Agricola, v.60, n.1, p. 77-82, 2003.

TRIGO, M.F.O.O.; TRIGO, L.F.N. Avaliação do vigor em sementes de pepino (Cucumis sativus L.). Informativo ABRATES, Londrina, v.5, n.2, p.131, 1995a.

TRIGO, M.F.O.O; TRIGO, L.F.N. Determinação da qualidade fisiológica de sementes de cenoura. Informativo ABRATES, Londrina, v.5, n.2, p.134, 1995 b. 